\title{
PERSPECTIVA COMO MEDIACIÓN ENTRE INTERPRETACIONES: HACIA UNA HERMENÉUTICA PERSPECTIVISTA DESDE LEIBNIZ ${ }^{1}$
}

\author{
Juan A. Nicolás \\ Universidad de Granada
}

\begin{abstract}
RESUMEN: En el presente artículo, me propongo abordar un concepto central del pensamiento de Heidegger, a saber, la noción de interpretación, a partir de la noción leibniziana de perspectiva, con la intenciónde señalar los posibles paralelismos entre dichas nociones. Por tanto, comenzaré con un análisis de los sentidos en que Leibniz emplea la noción de perspectiva y, a continuación pasaré a la noción del examen de la noción heideggeriana de interpretación. A partir de allí, examinaré la hipótesis del paralelismo entre las respectivas nociones y, como desarrollo de ello, la posibilidad de que la noción leibniziana de perspectiva sea apta para reconstruir la relación entre unidad y pluralidad no sólo en Leibniz, sino también en la hermenéutica actual.
\end{abstract}

PALABRAS CLAVE: Perspectivismo; punto de vista; hermenéutica; interpretación.

ABSTRACT: This article examines a central concept of Heidegger's thought - the notion of interpretation - from the point of view of the Leibnizian notion of perspective, in order to identify the possible parallels between these notions. I begin by analysing the different meanings Leibniz in which uses the notion of perspective; after that, I analyse Heidegger's notion of interpretation. On the basis of these analyses, I will examine the hypothesis of the existence of a parallelism between those two notions and therefore the possibility that Leibniz's notion of perspective could be used to explain the relationship between unity and plurality not only in Leibniz's own philosophy but also in contemporary hermeneutics.

KEYWORDS: Perspectivism; point of view; hermeneutics; interpretation.

\section{1. ¿Leibniz frente a Heidegger?}

No se intenta con esta reflexión analizar la relación histórica que en el plano filosófico pudo mantener Heidegger con Leibniz. De lo contrario el propio título de este apartado sería una simple anacronía. El objetivo es más

\footnotetext{
1 Este texto ha sido elaborado en el marco de las actividades del Proyecto "Leibniz en español" financiado por el Ministerio español de Economía y Competitividad (FFI2014-52089-P).
} 
bien avanzar en una confrontación sistemática entre estos dos filósofos de cara a la resolución de problemas filosóficos comunes hoy vigentes.

Así planteado, se trata de confrontar dos modelos filosóficos en la medida en que, salvadas las distancias cronológicas, sean confrontables y sean significativos los resultados de dicha confrontación. Que esta vía es transitable lo avala la abundante bibliografía que relaciona a ambos autores a propósito de muy variados temas, desde el principio de razón hasta el valor de la técnica o la evaluación de la ilustración ${ }^{2}$.

En concreto se trataría aquí de abordar el pensamiento de Heidegger desde la perspectiva de Leibniz, esto es, intentar contestar la pregunta acerca de qué diría Leibniz si tuviera la oportunidad de leer a Heidegger. Y todo ello referido a algún aspecto concreto de sus respectivas filosofías. Se intenta, pues, sacar rendimiento filosófico al pensamiento leibniziano en la actualidad.

La relación histórica de Heidegger con Leibniz fue más que notable, como atestiguan la multitud de textos dedicados por el primero a reflexionar sobre el pensamiento leibniziano a lo largo de prácticamente toda su vida activa. Se trata de dos filósofos tan inagotables el uno como el otro, y ambos han tenido una importante influencia en las posteridades respectivas.

Para abordar esta tarea se ha elegido una temática que no es de las más frecuentes en la literatura sobre ambos autores, pero que puede ser de gran

2 Sobre este aspecto puede verse Nicolás, "Perspektive und Interpretation. Leibniz und die Hermeneutik" (en prensa). 


\section{Dossiê Leibniz, Dissertatio - Volume Suplementar 03 I UFPel [2016]}

relevancia. Se trata de la noción de "perspectiva" y sus posibles paralelismos con la noción heideggeriana de "interpretación”.

Se plantea en primer lugar un análisis de los sentidos en que Leibniz utiliza la noción de "perspectiva" y sus respectivos alcances y ámbitos de aplicación. En segundo lugar, se analiza la tesis del carácter interpretativo de todo conocimiento en el marco de la transformación hermenéutica del saber. En tercer lugar, se plantea la hipótesis de un cierto paralelismo entre la noción leibniziana de perspectiva y la noción hermenéutica de interpretación. A continuación se examinan las hipótesis de comprender la perspectiva como interpretación, e inversamente, entender la interpretación como punto de vista o perspectiva.

Finalmente se plantea la hipótesis de que la noción de perspectiva, al ser siempre perspectiva de una misma realidad, según Leibniz, puede ser entendida como noción capaz de articular interpretaciones en un sistema a la vez plural y unificado. La noción de perspectiva se convertiría así en el concepto clave para reconstruir la relación entre unidad y pluralidad tanto en Leibniz como en la hermenéutica actual.

\section{Perspectiva e interpretación}

2.1. Plantea Leibniz que hay diversos modos de acceder al conocimiento de un objeto, de un hecho, de un problema o en general de una totalidad. Y que esos diversos modos de acceso pueden ser todos a la vez 
diferentes y verdaderos. Leibniz generaliza este rasgo como constitutivo del conocimiento humano y utiliza para designarlo la noción de "perspectiva". Todo conocimiento humano tiene carácter perspectivista, es un acercamiento a su objeto desde un determinado "punto de vista". No hay conocimiento humano exhaustivo de ningún objeto o hecho, siempre caben nuevos puntos de vista desde el que abordarlo (esto es, nuevas perspectivas) que ofrecerán nuevas informaciones. El requisito mínimo de las diversas perspectivas es el de la coherencia lógico-formal entre ellas.

Todo esto hay que situarlo en el marco de la epistemología leibniziana, en la que tiene un papel esencial la distinción entre punto de vista humano y punto de vista divino. He aquí dos "puntos de vista" inherentes a sus respectivos sujetos. El primer punto de vista representa el saber limitado humano, mientras que el punto de vista divino representa el saber absoluto. El saber absoluto consiste en la presencia intuitiva simultánea (atemporal) de la totalidad de las posibilidades en la mente divina. No se trata de una determinada perspectiva que es la verdadera, o más verdadera que las demás. Se trata del conjunto de la totalidad los puntos de vista parciales. O dicho de otro modo, se trata del conocimiento de la razón de la serie y de la serie de la razón. Esto se plantea también en relación con el subconjunto de posibilidades que constituye la combinación más potente de composibles que es la realidad.

La relación entre ambos puntos de vista ha de cumplir algunas características. En primer lugar, tener algo común, de lo contrario nada podría 


\section{Dossiê Leibniz, Dissertatio - Volume Suplementar 03 | UFPel [2016]}

saber el ser humano acerca de un punto de vista no humano, como sería el divino. En efecto, hay algunos principios de la razón que son válidos tanto para Dios como para el ser humano. Hay principios que "obligan" tanto a Dios como al ser humano, no pueden ser ignorados en un razonamiento válido o en un conocimiento verdadero. Esta comunidad entre ambos puntos de vista es lo que permite que el limitado saber humano pueda alcanzar parte del conocimiento absoluto de lo real y también de lo posible.

El carácter irrebasable de ciertos principios plantea al menos dos problemas: el problema de la libertad y el del carácter perspectivista del punto de vista absoluto.

(a) La pregunta por la libertad ha sido objeto de discusión desde el momento mismo de la formulación de la obra de Leibniz. Aquí sólo se pretende plantear un aspecto relacionado con el perspectivismo. El lugar de la libertad se problematiza especialmente en relación con los principios de la razón en el caso de la perspectiva absoluta o divina. Por ello es importante aclarar que para Leibniz la libertad no es (solamente) la posibilidad de elegir, sino que él maneja una concepción "ejecutiva" de libertad. Esta consiste en que la libertad tiene lugar cuando se realiza efectivamente la mejor opción. De este modo libertad es ser libre, y ser libre es actuar libremente, realizar la opción que nos hace libres, lo que nos libera. En el caso de Leibniz, esto consiste en actuar según la mejor razón. Dios es libre porque actúa según la mejor razón (principio de razón suficiente). La acción/decisión según la sabiduría (= razón) es la máximamente 
libre. He aquí la dimensión de la concepción leibniziana de libertad como liberación. Dios sería el ser libre (=liberado), en el caso del ser humano, este estado requiere un proceso de acercamiento y, a veces, de alejamiento, de carácter asintótico.

(b) Por otro lado, se plantea también, en relación con el punto de vista divino, la pregunta por su carácter perspectivista. Si se considera que el punto de vista absoluto es el conjunto de todos los posibles y cada uno de estos consiste en una combinación internamente coherente (composible) que entra a formar parte del conjunto de todos los posibles, cada mundo posible puede entenderse como un punto de vista determinado acerca de la totalidad de los posibles, es decir, como una perspectiva acerca del conjunto total. Si esta interpretación es viable en el marco leibniziano, puede considerarse que la estructura interna del punto de vista absoluto es perspectivista (la suma de todas las perspectivas lógicamente posibles). Ahora bien, el conjunto de todas las perspectivas no podría considerarse como una perspectiva, porque ésta por definición ha de ser limitada y parcial. En consecuencia, el punto de vista absoluto no es una perspectiva en ningún sentido, pero sí es perspectivista su estructura interna.

En segundo lugar, mientras el saber humano es principalmente discursivo y en cierta medida intuitivo, el saber del punto de vista absoluto ha de ser exclusivamente intuitivo. El carácter discursivo del saber humano lo sitúa en la perspectiva insondable del tiempo de modo que, en cuanto a contenidos o 


\section{Dossiê Leibniz, Dissertatio - Volume Suplementar 03 | UFPel [2016]}

a nuevas interpretaciones, no reconoce límite absoluto alguno. El límite formalmente irrebasable es el de la finitud, que Leibniz liga a la corporalidad ${ }^{3}$.

Esto significa que no hay verdad acerca de la cual no quepa un mayor avance, nuevos desarrollos, nuevas hipótesis y alternativas. Nada de esto afecta al punto de vista absoluto.

2.2. Por otro lado, Heidegger realiza una transformación en el modo de entender el conocimiento y el modo de estar en el mundo. Con ello ha fundado la hermenéutica contemporánea. En este planteamiento juega un papel fundamental la idea de que caben siempre diferentes aproximaciones a los hechos, a los objetos, a la verdad del conocimiento, al modo de entender el mundo y a nosotros mismos, etc. Y estos diversos modos de entender lo real y de estar en lo real pueden ser todos ellos parcialmente o totalmente verdaderos. Estas diversas aproximaciones a la verdad son entendidas como diversos modos de comprender o de interpretar los hechos, actos, objetos o sentimientos. De ahí que la noción de "interpretación" se convierte en central para la hermenéutica.

El concepto de "interpretación" ha de situarse, en el caso de Heidegger, en el marco de la tesis de que todo saber tiene lugar en una "situación hermenéutica". Ésta determina la pre-comprensión previa a la interpretación: "toda interpretación se funda en el comprender" ${ }^{4}$. El ser humano es originariamente un ser comprensor y la primera referencia de esta comprensión 
es el ámbito de la posibilidad: "El Dasein en cuanto comprender proyecta su ser hacia posibilidades. Este comprensor estar vuelto hacia posibilidades, por la repercusión que tienen sobre el Dasein esas mismas posibilidades en tanto que abiertas, es también un poder-ser. El proyectarse del comprender tiene su propia posibilidad de desarrollo. A este desarrollo del comprender lo llamamos interpretación" (HEIDEGGER, 2003, \32, p.172 - GA 2, 148).

Al igual que en el caso de Leibniz, a la base de la interpretación está el ámbito de lo posible. Sólo que en este caso, lo posible viene delimitado por el conjunto de determinaciones recogidas en el comprender. Respecto de ellas, la interpretación es un "desarrollo", una "articulación" o una "exposición”, por eso la interpretación consiste "en la elaboración de las posibilidades proyectadas en el comprender" (Ibid.).

El ámbito de la comprensión está constituido por todo el conjunto de supuestos estructurales de la acción interpretativo-cognoscitiva. Estos supuestos son organizados por Heidegger en tres planos distintos: haber previo (Vorbabe), manera previa de ver (Vorsicht) y manera previa de entender (Vorgriff) (HEIDEGGER, 2003, p. 248 - GA, 2, 232). Estos planos no están en el mismo nivel, sino que responden a una lógica de la constitución de la infraestructura del saber, con la que Heidegger pretende aclarar el método del comprender, esto es lograr "la transparencia metodológica del procedimiento comprensorinterpretativo de la interpretación del ser” (HEIDEGGER, 2003, \44, p. 246 GA, 2, 230). He aquí resumida la dinámica de los planos del comprender: "...el 


\section{Dossiê Leibniz, Dissertatio - Volume Suplementar 03 I UFPel [2016]}

haber previo, al cual deberán ajustarse todos los pasos ulteriores del análisis. Pero a la vez estos necesitan ser dirigidos por la posible manera previa de ver que apunta al modo de ser del ente en cuestión. Haber previo y manera previa de ver bosquejan entonces, al mismo tiempo, el repertorio de conceptos (manera de entender previa) a que deben ser elevadas todas las estructuras del ser" (HEIDEGGER, 2003, \45, p. 248 - GA, 2, 232). Esto significa que, de algún modo, toda la conceptualización que determina en último lugar la gama de interpretaciones posibles está en cierto tipo de dependencia respecto del haber previo y del modo previo de ver. Estos dos tipos de elementos son los que determinan propiamente las posibilidades esbozadas en el comprender preinterpretativo que culmina en la interpretación.

El proceso (estructural) de la comprensión-interpretación hace finalmente "comparecer" el mundo en términos de lenguaje y sentido (HEIDEGGER, 2003, \32, p. 170 - GA, 2, 151). No hay mera presencia no comprensivo-interpretativa, ni ante los sentidos ni ante el logos. He aquí un rasgo esencial del giro hermenéutico frente a Husserl y la comprensión fenomenológica del saber: "La interpretación no es jamás una aprehensión sin supuestos de algo dado" (HEIDEGGER, 2003, §32, p. 169 - GA, 2, 150) ${ }^{5}$. No cabe ni mayor impugnación de la fenomenología, ni mayor asentamiento del giro hermenéutico.

${ }^{5}$ Esta misma idea la expresa también con toda claridad del siguiente modo: "Toda simple visión antepredicativa de lo a la mano ya es en sí misma comprensora-interpretante" (§ 32, p.168 - GA 2, p.149). 
Todo saber (= comprender-interpretar) lo es siempre "en cuanto algo", es decir, en el marco de una determinada gama de posibles comprensiones e interpretaciones. El "paso" de esta gama de posibilidades (abierta y delimitada a la vez por el haber previo, Vorbabe) a una determinada interpretación (recortada por la manera previa de ver, Vorsicht) tiene lugar siempre "guiado por un punto de vista" (HEIDEGGER, 2003, \32, p. 169 - GA, 2, 150) que viene determinado en última instancia por la conceptualidad, esto es, por una manera de entender previa (Vorgriff). He aquí finalmente de nuevo la noción de punto de vista o "perspectiva".

En conclusión, en la Hermenéutica heideggeriana el saber tiene estructuralmente un carácter interpretativo, en cuyo fondo se halla siempre la determinación de un punto de vista. He aquí la estructura última de la concepción hermenéutica del saber, expresada por el propio Heidegger en términos perspectivistas. Se puede hablar sin forzar mucho las cosas de un perspectivismo de las interpretaciones.

Hasta aquí se han puesto de manifiesto suficientes paralelismos entre las nociones de "perspectiva" e "interpretación" como para formular la hipótesis de que el perspectivismo monadológico puede ser entendido como un interpretacionismo hermenéutico y a la vez la hermenéutica de las interpretaciones puede ser entendida como un perspectivismo.

Para comprender en detalle esta hipótesis y ponerla a prueba, conviene en primer lugar delimitar con rigor la noción leibniziana de "perspectiva". A 


\section{Dossiê Leibniz, Dissertatio - Volume Suplementar 03 | UFPel [2016]}

continuación habrá que descomponer el problema en sus ingredientes fundamentales y estructurar el análisis.

\section{La noción leibniziana de "perspectiva"}

La noción de perspectiva es clave en el pensamiento de Leibniz porque tiene un carácter transversal al menos en dos sentidos. Por un lado es utilizada en muy diversos ámbitos del saber, como se verá más adelante. Y por otro lado, está conectada con nociones fundamentales para la comprensión adecuada del pensamiento de Leibniz como las de orden, armonía, corporalidad, expresión, intersubjetividad (principe d'autrui), sustancia individual, belleza, totalidad y representación ${ }^{6}$.

Siendo así, se convierte en una tarea histórico-filosófica la reconstrucción rigurosa y detallada de esta noción en el pensamiento de Leibniz, tarea compleja por su extensión y profundidad, y que está aún por hacer. Se trata aquí de hacer una mínima aproximación a dicho quehacer.

Hagamos un breve repaso de lo que entiende Leibniz por "perspectiva". En primer lugar, hay que observar que desde el punto de vista semántico Leibniz utiliza a veces de modo equivalente las expresiones "perspectiva" y "punto de vista". Incluso a veces utiliza el giro "punto de perspectiva” (AA IV, 3, p. 903). Pero no siempre ocurre así.

${ }^{6}$ Cfr. Busche (1997). También Holze (2001). 
Como es habitual en Leibniz no hay un uso unívoco del término, sino que es utilizado en diversos sentidos y aplicado en diferentes ámbitos del saber. Se pueden distinguir al menos los siguientes sentidos del término perspectiva o punto de vista.

a) En primer lugar se entiende perspectiva como proyección. Esto ocurre en el frecuente uso que hace Leibniz de este término en el ámbito de la Geometría 7 .

Il est vray que la même chose peut être representée differemment; mais il doit toujours y avoir un rapport exact entre la representation et la chose, et par consequent entre les diferentes representations d'une même chose. Les projections de perspective, qui reviennent dans le cercle aux sections coniques, font voir qu'un meme cercle peut être representé par une ellipse, par une parabole et par une hyperbole... Rien ne paroit si different, ny si dissemblable, que ces figures; et cependant il y a un rapport exact de chaque point à chaque point (GP VI, p.327).

Es importante resaltar el hecho de que en esta acepción, todas las posibles perspectivas han de ser coherentes entre sí. La relación entre los elementos de las diferentes perspectivas proyectadas tiene carácter de expresión funcional:

Une chose exprime une autre, lors qu'il y a un rapport constant et reglé entre ce qui se peut dire de l'une et de l'autre, c'est ainsi qu'une projection de perspective exprime son geometral (AA II, 2, p.231) ${ }^{8}$.

b) En segundo lugar, Leibniz confiere al término perspectiva el sentido de referencia a las leyes de la visión humana. Las leyes de la perspectiva se entienden 


\section{Dossiê Leibniz, Dissertatio - Volume Suplementar 03 I UFPel [2016]}

aplicadas al ámbito del funcionamiento del ojo humano y al ámbito del arte. En el primer caso se refiere a la ciencia de la Óptica, y en el segundo caso se refiere principalmente a la pintura y los efectos producidos con el recurso a la perspectiva, y también a la arquitectura o la escultura. También se integra en este campo la reflexión sobre el arte que es la Estética.

Por un lado, el ojo tiene unas leyes de funcionamiento que en cierto modo "corrigen" lo representado. Es la dimensión biológica de la perspectiva. Esto da lugar a una serie de leyes científicas de la perspectiva. Por ejemplo, el ojo percibe simetría donde realmente no la hay 9 . El estudio y fijación de estas leyes constituye la Óptica ${ }^{10}$. Ésta tiene una rama de estudio de la trayectoria de los rayos de luz a través de un cristal o un espejo, y una parte de tallar vidrio para conseguir determinadas perspectivas y efectos ${ }^{11}$.

También en el ámbito del arte tienen una función las leyes de la perspectiva. En pintura es fundamental aplicar estas leyes para conseguir el efecto deseado y por parte del intérprete ha de estar también en la perspectiva correcta para captar el efecto adecuado ${ }^{12}$. Lo mismo ocurre en arquitectura (AA VI, 4, p. 2388) y en teatro, donde el juego de luces y sombras proyectadas desde diversos ángulos produce efectos especiales ${ }^{13}$

\footnotetext{
${ }^{9}$ AA IV, 3, p. 259; AA IV, 6, p.763.

${ }^{10}$ AA IV, 5, p.630; AA VI, 4, p.88.

${ }^{11}$ AA IV, 1, p.137 y 541; AA II, 1, p.263; AA VI, 4, p.1822.

${ }^{12}$ AA IV, 7, p. 219; AA II, 3, p.226; AA VI, 4, p.709; AA VI, 6, p.135.

${ }^{13}$ AA IV, 1, p.567-8; AA VI, 6, p.138.
} 
Finalmente, la perspectiva correctamente plasmada produce un efecto estético que es la belleza. Para ello es preciso estar en las circunstancias apropiadas ${ }^{14}$. Lo que aparentemente es feo o desagradable, desde la perspectiva adecuada muestra su belleza en el marco de la totalidad:

Dieu, par un art merveilleux, tourne tous les defauts de ces petits mondes au plus grand ornement de son grand monde. C'est comme dans ces inventions de perspective, où certains beaux desseins ne paroissent que confusion, jusqu'à ce qu'on les rapport à leur vray point de vue... Ainsi les deformities apparentes de nos petits mondes se reunissent en beautés dans le grand (GP VI, p.197-8).

c) Un tercer sentido del término perspectiva es como punto de vista diferenciado. En este tercer sentido es utilizado en el ámbito de la Metafísica, la Epistemología, la Ética y la Política.

En el terreno de la Moral y la Política Leibniz formula un principio que fija cuál es la perspectiva adecuada: "La place d'autruy est le vray point de perspective en politique aussi bien qu'en morale" (AA IV, 3, p.903) ${ }^{15}$. Situarse virtualmente en la posición (perspectiva) del otro es el mejor modo de comprenderlo, tanto si es para coincidir como para discrepar con él.

También en ciertos aspectos de la Ontología aparece la noción de perspectiva o punto de vista. Al determinar los componentes básicos de lo real Leibniz recurre a esta noción:

Il n'y a que les atomes de substance, c'est à dire, les unités reelles et absolument destituées de parties... On les pourroit appeller points

${ }_{14}^{14}$ AA II, 2, p.188; AA I, 13, p.13; AA I, 15, p.294-5.

${ }^{15}$ También AA IV, 1, p.79. 


\section{Dossiê Leibniz, Dissertatio - Volume Suplementar 03 U UFPel [2016]}

metaphysiques: ils ont quelque chose de vital et une espece de perception, et les points mathematiques sont leur points de vue (GP IV, 482-3).

Cada una de estas unidades ontológicas o sustancia individual representa un punto de vista propio y diferenciado. Se trata de una perspectiva acerca de la totalidad del mundo (AA II, 2, p. 188). "Les unités de substance n'estant autre chose que des diferentes concentrations de l'univers, representé selon les differens points de vue qui les distinguent" (GP IV, p.518). Se llega así al punto culminante del uso de la noción de perspectiva por parte de Leibniz. Los diferentes individuos se caracterizan por tener una perspectiva que los constituye como tal individuo diferenciado. Esta tesis tiene valor tanto ontológico (constituye individuos) como epistemológico (muestra el mundo de un determinado modo) ${ }^{16}$.

Puesto que hay infinitos individuos, hay otros tantos modos de acceder y expresar el mundo en su totalidad. Este es el famoso ejemplo utilizado por Leibniz:

Et comme une même ville regardée de differens cotés paroist tout autre et est comme multipliée perspectivement, il arrive de même, que par la multitude infinie des substances simples, il y a comme autant de differens univers, qui ne sont pourtant que les perspectives d'un seul selon les differens points de vue de chaque monade (Monadologie, GP IV, p. 616) ${ }^{17}$.

Todas las diferentes perspectivas tienen un doble principio de unificación: por un lado, están referidas a un único objeto, y por otro, están 
coordinadas según un plan profundo de orden, armonía y belleza instaurado por Dios. "Cette loy de l'ordre qui fait l'individualité de chaque substance particuliere, a un rapport exact à ce qui arrive dans tout autre substance, et dans l'univers tout entier"' (GP IV, p. 518).

Esta coordinación entre las diferentes perspectivas humanas verdaderas hace que no pueda haber perspectivas o puntos de vista incompatibles entre sí. No hay un punto de vista que sea el verdadero y los demás son falsos. Todos son relativamente verdaderos en la medida en que explican o representan parcialmente su objeto. Pero a su vez son intrínsecamente parciales, esto es, limitados; y esta limitación intrínseca está ligada a la corporalidad. Es la corporalidad la que constituye la perspectiva humana: "Dieu a fait l'ame en sorte qu'elle doit s'accorder avec tout ce qui est hors d'elle, et même le representer suivant les impressions que les choses font sur son corps organique, et qui fait son point de vue” (GP IV, p. 530).

El único "verdadero punto de vista" es el de la totalidad. La verdad es el todo, diría bastante más tarde Hegel. Pero no es ésta una perspectiva humana.

En conclusión pueden señalarse al menos tres características del perspectivismo leibniziano: diversas perspectivas tienen como referencia un objeto único; las diversas perspectivas están coordinadas entre sí, de modo que no puede haber puntos de vista verdaderos y absolutamente incompatibles; la limitación de cada perspectiva particular está ligada a la corporalidad. Estos tres rasgos están referidos al punto de vista humano. 


\section{Dossiê Leibniz, Dissertatio - Volume Suplementar 03 I UFPel [2016]}

\section{4. ¿Interpretación como perspectiva?}

Llegamos ahora al momento clave. Entre las nociones de 'interpretación" en el ámbito de la Hermenéutica y de "perspectiva" en el pensamiento leibniziano han ido apareciendo ciertos paralelismos evidentes. Que en sentido general una determinada perspectiva supone una cierta interpretación de lo conocido es evidente. Que una interpretación del mundo es acercarse a él desde un determinado punto de vista o perspectiva es también innegable. Se trata de dar un paso adelante en el rigor del análisis de estas tesis generales.

Para ello, la pregunta fundamental a plantear aquí no es si ha habido algún tipo de influencia histórica de Leibniz sobre Heidegger. Simplificando la cuestión, se puede decir que en un sentido laxo esta influencia ha tenido lugar sin duda a través de Nietzsche, a través de Hegel, y puede sospecharse, por ejemplo, detrás de la tesis común de que hay multitud de modos de acercamiento a la verdad de lo real. Pero este sentido laxo es filosóficamente casi irrelevante. Por otro lado, indagar esta posible influencia en un sentido histórico-crítico riguroso necesitaría un tiempo y un esfuerzo que no tienen aquí cabida. No es éste nuestro interés aquí.

Se trata más bien de hacer una confrontación sistemática entre estas dos nociones, independientemente de su conexión histórica real, que aquí dejaremos para otras investigaciones. 
En primer lugar habría que hacer una aclaración preliminar y rigurosa acerca de qué se entiende por interpretación, qué es hermenéutica, cómo conceptuar el perspectivismo y en qué contextos van a ser utilizadas estas nociones. Dado el carácter preliminar y metódico de estas definiciones, queden de momento en suspenso y pendientes de precisión, para que sea posible llegar al objetivo de esta reflexión.

¿Cómo pueden estas nociones de perspectiva e interpretación contribuir a un esclarecimiento del conocimiento humano? ¿Qué puede aportar cada una a la intelección de nuestro modo de estar en el mundo comprendiéndolo, esto es, con sentido? ¿Son nociones simplemente equivalentes o nada tienen que ver entre sí? ¿Hay elementos comunes entre estos dos modos de abordar y explicar el saber? ¿Cuáles son sus coincidencias y cuáles son sus divergencias? ¿Aportan algún rasgo relevante frente a otras concepciones filosóficas? ¿Se puede entender el carácter intrínsecamente interpretativo de la hermenéutica como un perspectivismo? ¿Puede entenderse el perspectivismo leibniziano como un modo "sui generis" de interpretacionismo? Todas estas son preguntas filosóficamente relevantes de cara a la construcción de una teoría de la intelección humana.

Abordarlas con cierto detalle constituye todo un programa de investigación. Me limitaré aquí solamente a esbozar lo que puede ser una línea de trabajo en el ámbito de los estudios leibnizianos, indicando sólo cómo podría plantearse sistemáticamente cada uno de los problemas. 


\section{Dossiê Leibniz, Dissertatio - Volume Suplementar 03 | UFPel [2016]}

Partamos de una sumaria caracterización del espíritu hermenéutico mediante dos rasgos esenciales: el giro hacia la facticidad como finitud (1) y la tesis de que "no hay hechos, sólo interpretaciones" (2).

1. Sobre el primer rasgo habría que dilucidar al menos dos cuestiones: en primer lugar, la prioridad ontológica de la posibilidad sobre la realidad (Leibniz) y la confrontación con el giro hacia la facticidad (Heidegger). Leibniz entiende la posibilidad en términos lógico-formales pre-existenciales; por el contrario, para Heidegger la gama de interpretaciones posibles viene determinada fácticamente por la estructura de la situación hermenéutica, es decir, pos-existencialmente. La analítica de la facticidad arroja como resultado la inmanencia, excluyendo todo rasgo absoluto. Heidegger establece la tesis de que realidad es contingencia. Leibniz por el contrario encuentra un momento de necesidad y absolutez en su analítica de lo real llevada a cabo mediante reflexión de la razón sobre sí misma (1.1.)

En segundo lugar, el papel que la infinitud (infinitum) juega en el análisis de la realidad en Leibniz y la confrontación con la equiparación hermenéutica de realidad y finitud (Heidegger). Frente al "ocasionalismo" de la finitud heideggeriano (HEIDEGGER, 1999, p. 25 - 1982, p.7), Leibniz incorpora el infinito en diversos momentos (divisibilidad de la materia, series de posibles, matemática, geometría, divinidad) de su planteamiento; infinito que puede ser conocido, pero "sólo confusamente". Aquí tendría cabida toda la discusión acerca del carácter discursivo del saber humano y junto con ello su historicidad. 
Puesto que según Leibniz nunca es posible llegar al conocimiento total ni siquiera de una simple noción de un sujeto individual (modelo asintótico), queda ahí patente la radical asunción por Leibniz de la finitud humana (1.2.)

2. En relación con la segunda tesis hay que atender a dos series de problemas. Primero, desde el punto de vista del sujeto, hay que abordar al menos dos cuestiones: (A) En Leibniz hay una doble perspectiva: la perspectiva humana, que siempre es relativa en ciertos aspectos, y la perspectiva divina, que representa un punto de vista absoluto. La limitación humana viene determinada por la corporalidad, que es a la vez posibilidad y límite, apertura y determinación. Todo perspectivismo humano es un perspectivismo corporal. Siendo así, tampoco para Leibniz hay hechos u objetos conocidos al margen de toda perspectiva (interpretadora). Sólo la postulación de un punto de vista no humano (absoluto) puede salvar esta limitación estructural del ser humano. En la Hermenéutica (Heidegger) es imposible la existencia de un punto de vista absoluto. Más bien hay una absolutización de la contingencia hasta convertirla en el único elemento irrebasable. Pero en la estructura que determina toda interpretación como finita está llamativamente ausente el elemento corporal (2.1.1.)

(B) También resulta disputable la relación de exterioridad o no entre el sujeto y su interpretación. Para Leibniz cada elemento de una perspectiva de un sujeto monádico es constitutivo de la propia mónada. Un sujeto individual es una determinada perspectiva acerca de la totalidad. El conjunto de cada uno de los 


\section{Dossiê Leibniz, Dissertatio - Volume Suplementar 03 I UFPel [2016]}

elementos constitutivos de una perspectiva es denominado por Leibniz "notio completa". Cambiar algo en esa noción significa constituir otro sujeto diferente.

Por el contrario, en una interpretación hermenéutica, la relación entre el interpretante y la interpretación no siempre tiene ese carácter constitutivo que tiene para Leibniz. En general, las interpretaciones tienen un carácter externo, aunque ciertamente, hay situaciones en que una determinada interpretación transforma al intérprete. He aquí otro elemento de confrontación (2.1.2.)

Segundo, desde el punto de vista del objeto del conocimiento, también habría que afrontar al menos dos cuestiones: (A) en la Hermenéutica es imposible alcanzar la 'cosa misma' dado el carácter constitutivamente interpretativo de la situación hermenéutica. En su versión más estándar, sólo es posible acceder a interpretaciones. Para Leibniz, por el contrario, todas las perspectivas lo son del mismo objeto. El objeto tiene prioridad ontológica para Leibniz respecto a su conocimiento del mismo. ¿Cómo ha de entenderse esto? ¿Cómo es posible saber que efectivamente, diversas perspectivas lo son de un mismo objeto? ¿Hay un acceso (en algún sentido) al objeto no perspectivista?¿Se trata de un mero realismo injustificado por parte de Leibniz? (2.2.1.)

(B) De ahí se deriva la siguiente cuestión: ¿Hay interpretaciones y perspectivas mejores que otras? Se abre con ello la pregunta criticista lanzada tanto contra el presunto realismo leibniziano como contra la hermenéutica no normativa y desfundamentadora. ¿Hay criterio "universal” para valorar comparativamente perspectivas e interpretaciones diferentes? ¿Cuál puede ser la 
fuente de dicho criterio discriminador? El criterio puede proceder de la cosa misma (Leibniz) o del "haber previo" (Vorhabe, Heidegger). Leibniz propone precisamente como tarea del pensamiento para cada individuo descubrir el orden general en que está inmerso, porque "non seulement tout va par ordre, mais mesme nos esprits doivent 'en appercevoir de plus en plus à mesure qu'ils font des progrés” (GP VII, 543) ${ }^{18}$. El criterio para la crítica se sitúa en el “veritable point de vue des choses" (GRUA 380).

Queda así planteada una última cuestión que puede ser filosóficamente relevante para la filosofía actual (2.2.2.)

He aquí sistemáticamente planteado un modo de llevar adelante una investigación acerca de la aportación del perspectivismo leibniziano a una discusión, crítica y desarrollo de la hermenéutica actual.

\section{Perspectiva como mediación entre interpretaciones}

En el ámbito de la hermenéutica filosófica existe una larga y enconada polémica acerca de la posibilidad de fundamentación del saber. Esta polémica no sólo comprende disputas, por ejemplo, entre Apel y Vattimo o entre Habermas y Derrida. Yendo a la raíz histórica cercana de la génesis de la hermenéutica que representa Heidegger, también ahí pueden encontrarse posiciones muy divergentes en lo relativo a la posibilidad de fundamentación. Hay interpretaciones abiertamente no normativas del pensamiento

\footnotetext{
18 Sobre la noción leibniziana de "orden" puede verse Rauzy (1995).
} 


\section{Dossiê Leibniz, Dissertatio - Volume Suplementar 03 I UFPel [2016]}

heideggeriano, basadas principalmente en el segundo Heidegger, y hay también reconstrucciones del pensamiento heideggeriano que otorgan un lugar central a cierto tipo de normatividad ${ }^{19}$.

Tras la reflexión hasta aquí desarrollada, puede lanzarse la hipótesis de que la noción de 'perspectiva' acuñada por Leibniz puede jugar un papel relevante para comprender la dinámica de las interpretaciones, entendidas como puntos de vista diferentes, manteniendo a la vez la posibilidad siempre de nuevas interpretaciones, pero sin perder una lógica interna que las unifica sistemáticamente. De este modo se rebasa la concepción de la verdad única en cada caso, pero manteniendo una referencia ordenadora, esto es, permaneciendo dentro del marco de un cierto tipo de racionalidad. Así entendido, puede decirse que Leibniz elabora una especie de transformación de la racionalidad en términos de bermenéutica perspectivista. Y eso a su vez, puede volverse críticamente contra las hermenéuticas no normativas, aportando argumentos en favor de la posibilidad de fundamentación dentro de este tipo de planteamiento, esto es, a favor de un cierto tipo de hermenéutica crítica.

La noción de perspectiva es un intento por parte de Leibniz de recoger todas aquellas circunstancias que tienen lugar en el acto del conocimiento implantado en la realidad,

permite al ser humano atenerse a su experiencia y a su perspectiva, y a la vez estar dispuesto a reconocer su falibilidad. En última instancia permite que la perspectiva cumpla su función de facilitar la incorporación del sujeto a la vida (SALAS, 1995, p. 111).

${ }^{19}$ Sobre este asunto puede verse Delgado e Nicolás (2015). 
De ahí el lugar preferente otorgado a la corporalidad y con ello a la percepción, que Leibniz estira hasta la apercepción más allá de la conciencia ${ }^{20}$. De este modo, Leibniz rebasa la reducción del saber a lógica del conocimiento (Racionalismo crítico) y rebasa los epistemologicismos. La perspectiva del sujeto individual viene condicionada de antemano por el objeto mismo, el individuo pertenece a un sistema en el que cada uno tiene su propia ley interna, que forma parte de una legislación universal anterior a cada individuo: "Chaque monade est un miroir vivant... representatif de l'univers, suivant son point de vue, et aussi reglé que l’univers luy même” (GP VI, p. 599.)

En este planteamiento el perspectivismo leibniziano no constituye un relativismo de los puntos de vista (o de las interpretaciones). No toda interpretación es posible, porque cada individuo pertenece a un mundo común regido por leyes, reglas y principios que establecen el orden general de la razón (perspectivista) (BOUQUIAUX, p. 52). Cada individuo es lo que es en relación a todo el resto del sistema, que a su vez tiene una estructura dinámica en la que la función y alcance de cada elemento (individuos, principios, leyes) es variable. En esta dinámica, la perspectiva de cada individuo es constitutiva de su ser, no es algo externo; por ello no es posible separar la dimensión ontológica de la dimensión epistemológica en el perspectivismo. No se trata de que cada individuo tiene una esencia propia y además una determinada posición respecto

20 Un intento actual de aprovechamiento de la noción leibniziana de corporalidad en un contexto hermenéutico puede verse en Apel (1975). 


\section{Dossiê Leibniz, Dissertatio - Volume Suplementar 03 | UFPel [2016]}

a todo lo demás, sino que cada individuo es su facticidad lógico-corporal. Cada individuo es perceptio y appetitus dentro de un sistema dinámico que determina sus posibilidades, y por tanto, su realidad. La permanente transformación de las perspectivas constituye proceso continuo (principio de continuidad) de unas representaciones a otras, de unas percepciones a otras, de lo confuso a lo claro, de lo ignoto a la luz de nuevas interpretaciones. Un ejemplo es la propuesta heliocéntrica introducida por Copérnico. Esta conexión universal de todo con todo ("tout est lié") es un principio esencial para Leibniz: "L'action de chacune se conserve dans chacune de tout l'univers à cause de la conspiration et sympathie de toutes choses, le monde estant tout entier dans chacune de ses parties, mais plus distinctement dans les unes que dans les autres" (GP VII, p. $544)$.

La noción de perspectiva es la que permite moverse por el espacio lógico y ontológico. De ahí que "quien conoce uno de mis principios los conoce todos". Esta noción permite acceder a la totalidad desde cada individualidad y con ello conjugar unidad y pluralidad, individualidad y totalidad, verdad y diversidad. Dinámicamente concebida, la noción de perspectiva se constituye en la "argamasa" teórica que permite mantener la infinita diferencia dentro de un orden, sin que aquélla se pierda en la unidad indiferenciada, permite en definitiva, que la irreductible diversidad pueda seguir siendo concebida como sistema. 
He aquí una reconstrucción del modelo perspectivista que propone Leibniz, que puede entenderse en términos de hermenéutica porque no viene determinado por unos principios a priori, sino que las cosas son lo que son en cada contexto, en cada ocasión, en cada lugar del sistema. Cada perspectiva expresa todo el orden general solidariamente, esto es, desde la conexión de todo con todo, y a su vez, cada perspectiva es lo que es en virtud de sus relaciones con lo demás. El modelo perspectivista leibniziano expresa que toda verdad es relativamente relativa, y no absolutamente relativa. Así planteado, uno de los méritos de Leibniz quizás radique en que ese "giro a la facticidad" que practica no lo conduce a la imposiblidad de fundamentar el saber. Esta vía leibniziana es la que puede resultar fructífera en su confrontación con otros modelos de filosofía hermenéutica hoy vigentes.

\section{BIBLIOGRAFIA}

APEL, K. O. "Das Leibapriori der Erkenntnis. Eine Erkenntnisanthropologische Betrachtung im Anschluss an Leibnizens Monadenlehre“. In GADAMER, H.G. VOGLER, P. (edits.): Neue Anthropologie. Stuttgart, Munchen, 1975, Bd. 7, p. 264-288.

BOUQUIAUX, L. "La notion de point de vue dans l'élaboration de la métaphysique leibnizienne". In TIMMERMANS, B. (coord.). Perspective. Leibniæ, Whitehead, Deleure. París: Vrin, 2006. 


\section{Dossiê Leibniz, Dissertatio - Volume Suplementar 03 | UFPel [2016]}

BUSCHE, H. Leibniz'Weg in perspektivische Universum. Eine Harmonie im Zeitalter der Berechnung. Hamburg: Meiner Verlag, 1997.

GÓMEZ DELGADO, J. M., NICOLÁS, J.A. “Hermenéutica y normatividad: interpretaciones de la filosofía de Heidegger”. Diálogo Filosófico, 81, 2015, p. 437.

HEIDEGGER, M. Ontología. Hermenéutica de la facticidad. Madrid: Alianza, 1999.

. Ontologie. Hermeneutik der Faktizität, V. Klostermann,

Frankfurt am Main, 1982.

Ser y Tiempo. Rivera, J.E. (trad.). Madrid: Ed. Trotta, 2003.

HEIDEGGER, M. (GA) Gesamtausgabe. Vittorio Klostermann Verlag.

HOLZE, E. E. Holze, "Mensch, Perspektive, Gott.

Leibniz'Perspektivitätstheorie als neuzeitliches Pluralismusmodell’. In VII.

Internationaler Leibniz-Kongress. POSER, H (Hrsg.). Hannover, 2001, p. 516523.

NICOLÁS, J. A. "Gnoseología del perspectivismo corporal en Leibniz”. Cuadernos Salmantinos de Filosofia, 40, 2013, p. 135-150.

RAUZY, J.-B. "Quid sit natura prius? La conception leibizienne de l'ordre”. Revue de Métaphysique et de Morale, 100, 1995, p. 31-48.

SALAS, J. "Perspectiva y contingencia en Leibniz". Anuario Filosófico, 38, 1995. 